\title{
Energy harvesting from organic liquids in micro-sized microbial fuel cells
}

\author{
Justine E Mink ${ }^{1,2}$, Ramy M Qaisi ${ }^{1}$, Bruce E Logan ${ }^{3}$ and Muhammad M Hussain ${ }^{1}$
}

Micro-sized microbial fuel cells (MFCs) are miniature energy harvesters that use bacteria to convert biomass from liquids into usable power. The key challenge is transitioning laboratory test beds into devices capable of producing high power using readily available fuel sources. Here, we present a pragmatic step toward advancing MFC applications through the fabrication of a uniquely mobile and inexpensive micro-sized device that can be fueled with human saliva. The $25-\mu l$ MFC was fabricated with graphene, a two-dimensional atomic crystal-structured material, as an anode for efficient current generation and with an air cathode for enabling the use of the oxygen present in air, making its operation completely mobile and free of the need for laboratory chemicals. With saliva as a fuel, the device produced higher current densities $\left(1190 \mathrm{Am}^{-3}\right)$ than any previous aircathode micro-sized MFCs. The use of the graphene anode generated 40 times more power than that possible using a carbon cloth anode. Additional tests were performed using acetate, a conventional organic material, at high organic loadings that were comparable to those in saliva, and the results demonstrated a linear relationship between the organic loading and current. These findings open the door to saliva-powered applications of this fuel cell technology for Lab-on-a-Chip devices or portable point-of-care diagnostic devices. NPG Asia Materials (2014) 6, e89; doi:10.1038/am.2014.1; published online 7 March 2014

Keywords: graphene; microbial fuel cell; saliva

\section{INTRODUCTION}

Microbial fuel cells (MFCs) rely upon the decomposition of organic matter by bacteria, which in turn transfers electrons to the anode. Electrons are then delivered through an external load to the cathode to produce a current. ${ }^{1,2}$ Although one of the most unique advantages of MFCs is that they can be used to both clean water and produce electrical power, the objective of the micro-sized MFC is to maximize power production for use as viable liquid-fueled miniature energy harvesters. ${ }^{3,4}$ Because micro-sized MFCs utilize less electrode area and less liquid fuel volume than their macro-sized counterparts, optimizing the electrodes and the fuel sources are the most important factors in designing a micro-sized MFC for maximum power production. Therefore, the selection of the electrode material is crucial. Other requirements of an ideal micro-sized MFC device include the following: an anode with a high surface area-to-volume ratio to enhance the collection of electrons from the bacteria; an air cathode integrated into a single-chamber MFC to lower the internal resistance of the entire system; and device mobility, as it does not require the use of unsustainable chemicals at the cathode. Another important aspect of an ideal micro-sized MFC is the ability to utilize an abundant and concentrated fuel source that can generate high power outputs with minimal liquid volumes. We therefore developed a micro-sized MFC that contained an anode made of the carbon nanomaterial graphene and an air cathode, and we powered this MFC using human saliva (Figure 1).

\section{MATERIALS AND METHODS}

Multi-layer graphene anode

The materials and configurations of the anode and cathode were optimized to increase power production using a $25-\mu \mathrm{l}$ MFC. Electrodes require high conductivity, chemical stability, corrosion resistivity and high mechanical strength. ${ }^{1,5}$ Because the anode must also support the growth of bacteria, it must also have good biocompatibility. Enhanced anodes have high surface-tovolume ratios to provide the maximum possible surface area for bacteria, ${ }^{6}$ as well as excellent electrical properties, to enable efficient electron transfer. ${ }^{7}$

Anode materials are usually carbon based. ${ }^{8}$ Due to the exceptional electrical and structural properties of nano-engineered carbon materials, such as carbon nanotubes (CNTs) and, most recently, graphene oxides, studies have incorporated these materials into MFC anodes. ${ }^{7,9-12}$ The specific surface areas of CNTs and the two-dimensional atomic crystal-structured material graphene are at least three orders of magnitude $\left(10^{3}\right)$ higher than that of conventional carbon-based electrodes $\left(<1 \mathrm{~m}^{2} \mathrm{~g}^{-1}\right)$. Furthermore, these materials have intrinsic high electrical conductivities $\left(>10 \mathrm{~S} \mathrm{~cm}^{-1}\right)$,

${ }^{1}$ Integrated Nanotechnology Lab, Electrical Engineering Program, Computer, Electrical, Mathematical Science and Engineering Division, King Abdullah University of Science and Technology, Thuwal, Saudi Arabia; ${ }^{2}$ Water Desalination and Reuse Center, Environmental Science and Engineering Program, Biological and Environmental Science and Engineering Division, King Abdullah University of Science and Technology, Thuwal, Saudi Arabia and ${ }^{3}$ Department of Civil and Environmental Engineering, The Pennsylvania State University, University Park, PA, USA

Correspondence: Dr MM Hussain, Integrated Nanotechnology Lab, Electrical Engineering Program, Computer, Electrical, Mathematical Science and Engineering Division, King Abdullah University of Science and Technology, Building 3 (Ibn Sina), Level 3, Room No. 3.274, Thuwal, Makkah 23955-6900, Saudi Arabia.

E-mail: muhammadmustafa.hussain@kaust.edu.sa

Received 4 August 2013; revised 24 November 2013; accepted 26 November 2013 


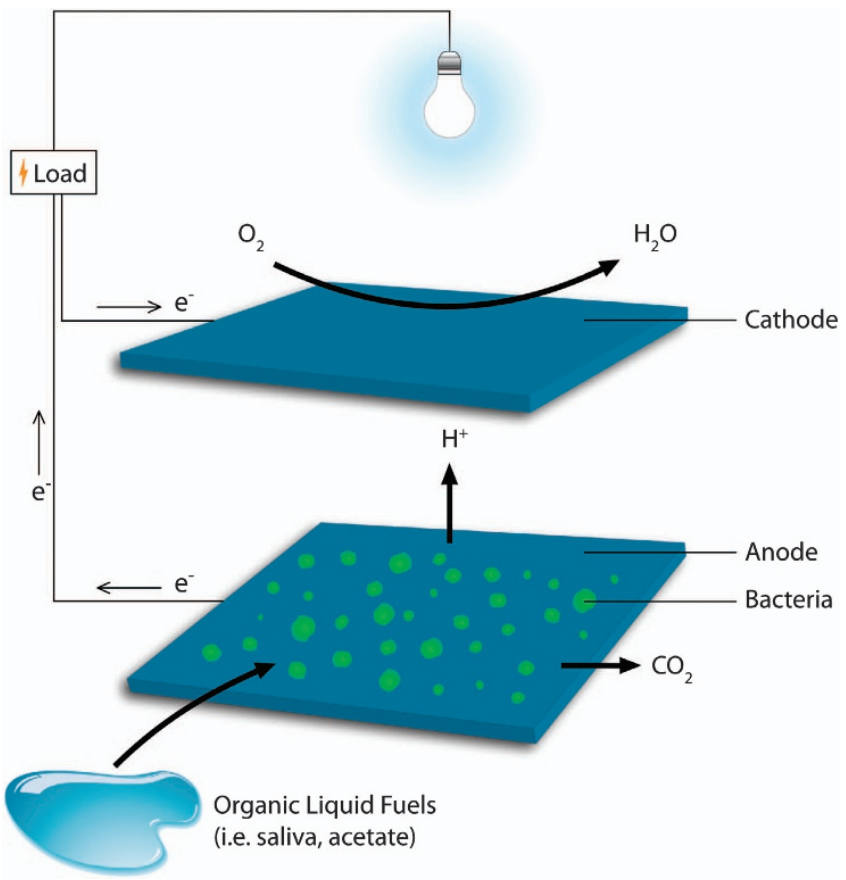

Figure $1 \mathrm{MFC}$ process, schematic and digital photographs of the microsized MFC. MFC process diagram describing the main components of an MFC and reactions occurring within the device. Organic liquids are broken down by bacteria into protons, electrons and carbon dioxide at the anode, and oxygen from the air is utilized at the cathode.

potentially making them ideal anode materials to enhance power densities. ${ }^{13}$ Most studies have integrated CNTs into existing anode materials, such as carbon cloth ${ }^{10}$ or textiles. ${ }^{11}$ In our previous work, we used multi-walled CNTs as an anode to produce high current density MFCs. ${ }^{12}$ Recently, graphene oxide has also been introduced as a blended anode material in carbon cloth ${ }^{14-16}$ and stainless steel. ${ }^{17}$ We show here, for the first time in any type of MFC, that anodes can be composed of pure multi-layer graphene rather than a graphene oxide hybrid. To produce these anodes, multi-layer graphene was synthesized via the decomposition of methane over copper using atmospheric pressure chemical vapor deposition. First, $25-\mu \mathrm{m}$ thick copper foils (99.8\%, Alfa Aesar, Karlsruhe, Germany) were loaded into a 4-inch quartz tube inside a horizontal furnace. The system was then purged with nitrogen for $5 \mathrm{~min}$ to remove any impurities inside the tube. Then the system temperature was ramped to $1000{ }^{\circ} \mathrm{C}$ in $30 \mathrm{~min}$ while argon and hydrogen were flowed through the quartz tube at a rate of 200 and 500 standard cubic centimeters per minute (s.c.c.m.), respectively, for the initial cleaning of $\mathrm{Cu}$. The sample was then kept under 500 s.c.c.m. of hydrogen for $4 \mathrm{~min}$ to remove oxygen and to stabilize the surfaces of the $\mathrm{Cu}$ foils. Methane gas, at a flow rate of 50 s.c.c.m., was introduced into the tube for $1 \mathrm{~min}$. Thereafter, the growth was terminated, and the furnace was cooled to room temperature under a flow of nitrogen.

Raman spectroscopy ( $532 \mathrm{~nm}$ laser wavelength) was used to evaluate the multi-layer graphene synthesized using the aforementioned process. Graphene was confirmed by optical images and the corresponding Raman spectrum (Supplementary Figure S1).

\section{Air cathode}

Micro-sized MFCs are often constrained by their reliance on a two-chamber configuration, in which the cathode and anode are separated by a membrane and the cathode chamber must be refilled with fresh electron acceptors. Ferricyanide is almost exclusively used in micro-sized MFCs as an electron acceptor, because it can generally produce higher power densities than more readily available electron acceptors, such as oxygen, because of its ability to maintain the cathode potential closer to the theoretical value. ${ }^{5}$ Ferricyanide must be continuously refilled, however, making it an unsustainable design feature for use outside of the laboratory. ${ }^{1,5}$ Thus, the use of the oxygen present in air has been explored as an electron acceptor, as it is readily available and does not need to be regenerated. Air cathodes, which use ambient oxygen as the electron acceptor, are commonly used in large-scale MFCs, which typically contain a hydrophobic carbon/polytetrafluoroethylene layer on the air side of the cathode to prevent water leakage and control oxygen diffusion, as well as a thin platinum catalyst on the water side, as described previously. ${ }^{18,19}$ However, air cathodes have only just recently been introduced into micro-sized MFCs and usually possess proton exchange membranes that are hot pressed onto a carbon cloth cathode. ${ }^{20,21}$ Membranes, however, increase the cost and the internal resistance of a device, which lowers the overall power production. ${ }^{5,18}$ Therefore, we designed a single-chamber, membrane-less, micro-sized MFC with an air cathode, which makes the device completely mobile as it does not require any chemicals other than the oxygen present in air.

\section{MFC assembly and operation}

The graphene foil was cut to form a square $(1 \mathrm{~cm} \times 1 \mathrm{~cm})$. The rubber spacer ( $1 \mathrm{~mm}$ thick) used between the cathode and the anode was cut to the same size, and a square hole $\left(5 \times 5 \mathrm{~mm}^{2}\right)$ was cut into the spacer center to function as the anode chamber. Syringe tips were inserted into both sides of the rubber to serve as the inlet and outlet for feeding or inoculation of the anode. The graphene foil anode was glued to one side of the rubber spacer, and the $1 \times 1 \mathrm{~cm}^{2}$ cathode was glued to the other side. This assembly was adjusted to leave one side of the spacer slightly off the center of the anode such that enough graphene foil was exposed to allow for contact with the anode. Another piece of the rubber was glued to the bottom of the anode as a support for the thin foil anode. Space was left on the contact side so that an alligator clip could be directly attached to the spacer-anode to contact the graphene on the anode. A second clip was attached to the air cathode to provide contact to the cathode. Both clips were first attached to a $1000 \Omega$ resistor and then to a multimeter (Keithley, Cleveland, $\mathrm{OH}, \mathrm{USA}$ ) to record the voltage every $20 \mathrm{~min}$. The same set-up procedure was performed by substituting a carbon cloth anode for the graphene foil. The devices were inoculated with waste water from the local waste water treatment plant primary clarifier, and after two separate inoculations, the solutions were switched to the fuels being tested (saliva or acetate). Further details on the saliva are provided below. Different concentrations of acetate were added to a $25 \mathrm{~mm}$ phosphate buffer solution $\left(\mathrm{NH}_{4} \mathrm{Cl}, \mathrm{NaH}_{2} \mathrm{PO}_{4} \bullet \mathrm{H}_{2} \mathrm{O}, \mathrm{Na}_{2} \mathrm{HPO}_{4} \bullet \mathrm{H}_{2} \mathrm{O}\right.$ and $\left.\mathrm{KCl}\right)$ with vitamins and minerals, as described previously. ${ }^{22}$

The micro-sized MFC system created here has a bottom support and liquid chamber that are both composed of inexpensive flexible rubber, making the MFC bendable and inexpensive (Figure 2). The flexible rubber structure can be attached to a variety of surfaces and can be easily produced without difficulty, making the entire device easily deployable.

\section{Use of saliva as fuel}

We utilized this graphene anode/air cathode micro-sized MFC to test whether human saliva could be a viable fuel source for powering such a device. Although predominantly water, saliva is composed of inorganic and organic components, such as glucose, that can be utilized for fuel by bacteria. ${ }^{23}$ Saliva was collected from the same individual (a healthy 16-year-old) at the same time every day, with food and drink being withheld for at least an hour beforehand, as described in typical salvia collection protocols. ${ }^{23}$ Sufficient saliva was collected to perform a chemical oxygen demand analysis for each sample, with organic loadings between $13400-15500 \mathrm{mgl}^{-1}$, a conductivity of $2.2-2.8 \mathrm{mS} \mathrm{cm}^{-1}$ and a $\mathrm{pH}$ of 7 . Bacteria were first inoculated into the device using wastewater (primary clarifier from a local wastewater treatment plant) before the saliva samples were inserted (same saliva for each device). Because the objective of the experiment was to produce a device that could be operated under commonly encountered conditions, there is a natural variability due to the bacterial inoculation that is present in the wastewater; thus the actual saliva was utilized rather than a synthetic fuel.

\section{Energy losses}

The overall energy loss in an MFC system can be estimated using:

$$
V_{\text {device }}=O C V-I R_{\text {internal }}=O C V-I\left(R_{a}+R_{c}+R_{m}+R_{e}\right)
$$



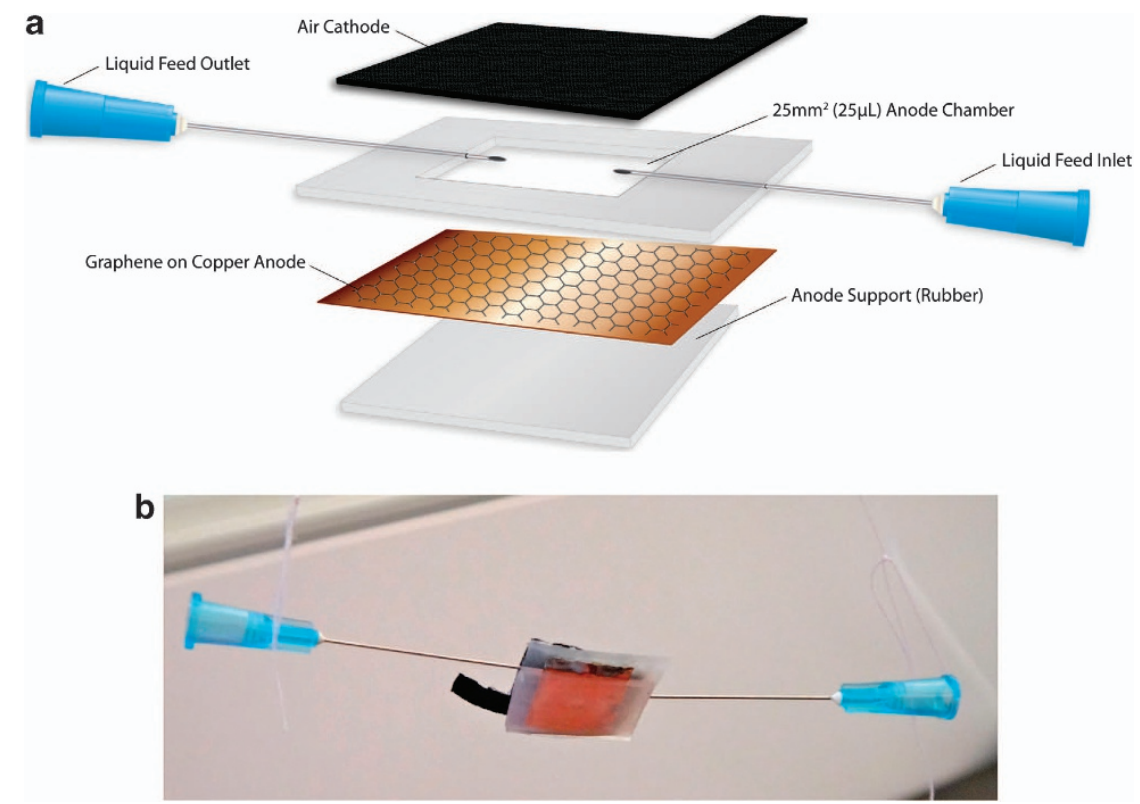

Figure 2 Micro-sized MFC schematic and digital photographs. (a) Stacked architecture of the $25-\mu l$ micro-sized MFC with inexpensive rubber support structures, sustainable air cathode and graphene anode. The single-chamber configuration is unique in micro-sized MFCs, as it lacks a membrane, which improved power production by avoiding internal resistance loss produced by a membrane. (b) The multi-layer graphene anode was grown on top of the copper foil and sandwiched in rubber, enabling the high flexibility of the device. The exceptional properties of graphene, particularly its increased surface area and high conductivity, resulted in substantially improved power production.

where the voltage of the device is equal to the difference between the open circuit voltage $(O C V)$ and the maximum current $(I)$ times the internal resistance $\left(R_{\text {internal }}\right)$, which is composed of resistances from the anode $\left(R_{a}\right)$, cathode $\left(R_{c}\right)$, membrane $\left(R_{m}\right)$ and electrolyte used $\left(R_{e}\right)$. The entire energy loss of the graphene system was approximately $110 \mathrm{mV}(\mathrm{OCV} 220-90 \mathrm{mV}$ at maximum power) and $53 \mathrm{mV}(O C V 119-66 \mathrm{mV}$ at maximum power) for the carbon cloth. Because there is no membrane in our system, an entire source of resistance is eliminated $\left(R_{m}\right)$. The electrolyte or solution resistance can be estimated using $R_{e}=d /(A K)$, where $d$ is the electrode distance $(\mathrm{cm}), A$ is the geometric area available for ionic species to pass $\left(\mathrm{cm}^{2}\right)$ and $K$ is the specific conductivity $\left(\Omega^{-1} \mathrm{~cm}^{-1}\right)$ of the solution. The distance between the anode and the cathode is approximately $0.1 \mathrm{~cm}$ over an area of $5 \times 5 \mathrm{~mm}^{2}$ in a saliva solution, with a conductivity of approximately $0.0022 \Omega^{-1} \mathrm{~cm}^{-1}$.

\section{RESULTS}

As shown from the current cycles over time in Figure 3a, with the introduction of saliva, the current cycles for the graphene anode first peaked at $130 \mathrm{Am}^{-3}$ and then increased to $1190 \mathrm{Am}^{-3}$ with subsequent fuelling cycles. At a current density of $1190 \mathrm{~A} \mathrm{~m}^{-3}$, the graphene anode MFC produced higher current densities than any other previous air cathode micro-sized MFCs, as shown in Supplementary Table S1.

We also tested the performance of saliva with a commonly used MFC anode, carbon cloth; with the exception of the anode, the other conditions were identical. The maximum peak for the carbon cloth device was $770 \mathrm{Am}^{-3}$. Therefore, with only saliva as a fuel, the graphene anode outperformed the carbon cloth by 55\%. From the power density curve (Figure $3 b$ ), graphene generated higher power $(60 \times)$ and current densities $(40 \times)$ than that obtained with the carbon cloth, confirming the hypothesis that saliva could power micro-sized MFC devices and demonstrating the enhanced performance of the graphene anode.

One of the reasons graphene outperformed the carbon cloth is that graphene has been found to increase the electron transfer kinetics of the anode and enhance the catalytic activity of oxidation, increasing the power produced by graphene-enriched MFC anodes. ${ }^{24}$ Specifically, cyclic voltammogram plots and electrical impedance spectroscopy were used to characterize a graphene biofilm to show that graphene improved the kinetics of electron transfer between the cell surface proteins and the electrode, leading to an overall improvement in electron transfer kinetics. ${ }^{16,17,24}$

An analysis of energy loss was performed to determine the specific contributions of the various components of the device to the total internal resistance of the system. The electrolyte resistance was nearly $180 \Omega$; therefore, using the maximum power, a current of $1.4 \mu \mathrm{A}$ results in a loss of approximately $0.252 \mathrm{mV}$. By calculating the energy losses in the graphene and carbon cloth systems, we could obtain a better understanding of the maximum potential energy output under these conditions, as well as the probable causes of energy loss in these systems. The components of energy loss include the calculated resistances for the anode, cathode, membrane and fuel source. Membrane resistance is eliminated in our single-chamber system, and the saliva resistance was minimal, leaving the electrodes as the major source of energy loss in the devices. As the cathodes were the same for both the graphene and carbon cloth devices, the major contributor to the difference in energy loss between the systems was the anode. Although the graphene anode device had a higher $O C V$, and therefore a higher potential maximum power production $(220 \mathrm{mV}$ for graphene, $119 \mathrm{mV}$ for carbon cloth), the energy loss in the system was $50 \%$ compared to only $44 \%$ for carbon cloth. We speculate that a larger portion of the difference in energy loss results from contact engineering issues, which are natural in a low-density-of-state material, such as graphene. There are additional contact challenges associated with the difficulty in depositing electrodes directly onto graphene because of the low interaction energy between the metal and carbon atoms. ${ }^{25}$ 

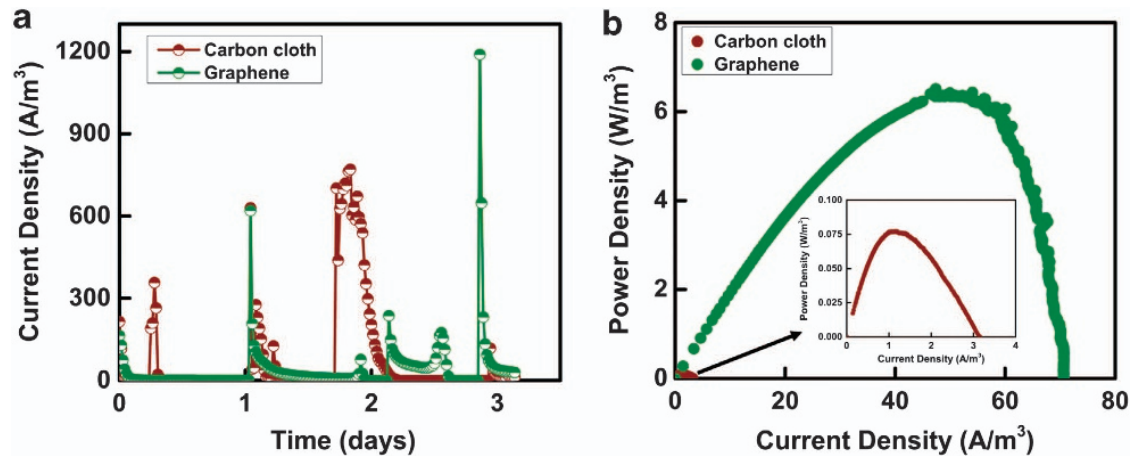

Figure 3 Current and power generation with saliva. (a) Current production, following startup, with saliva, using graphene or carbon cloth anodes, where each peak in current reflects the refueling with saliva. (The initial first two cycles of bacterial inoculation are not shown.) The maximum peak for the graphene anode was $55 \%$ higher than that obtained with the carbon cloth. (b) Power density, demonstrating graphene outperforming carbon cloth, with $60 \times$ more power and $40 \times$ higher in terms of maximum current density. The inset shows the peak for carbon cloth at $<0.1 \mathrm{Wm}^{-3}$.
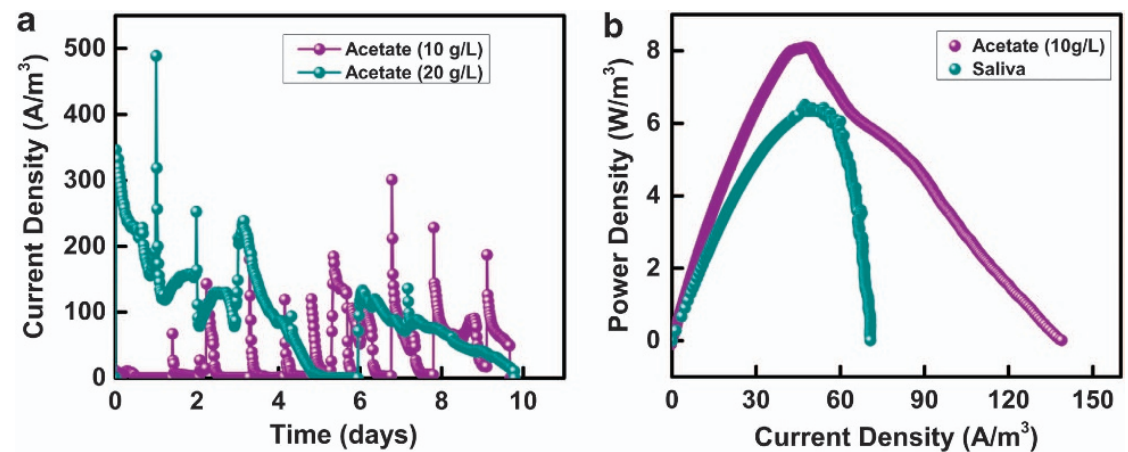

Figure 4 Current and power generation with high acetate loadings. (a) We tested the $25-\mu l \mu M F C$ with high concentrations of a conventional fuel source (acetate at 20 and $10 \mathrm{gl}^{-1}$ ). The $20-\mathrm{gl}^{-1}$ acetate device outperformed the $10 \mathrm{-g} \mathrm{I}^{-1}$ device with peaks at 12.2 and $7.5 \mu \mathrm{A}$, respectively. (b) Power density curves were also measured for the devices and compared with those of the nonconventional organic source, saliva. Higher power production by the acetate device, although at a lower organic loading, was likely due to either a larger variety of organic materials in saliva that were more difficult for the bacteria to break down or a slightly higher conductivity in the acetate solution. Power density curves could not be obtained for the $20-\left.\mathrm{g}\right|^{-1}$ device, however, because by the time the measurements were performed (after the device had been operated for almost 10 days), the high acetate content was visible as a powder and clogged the feeding syringes of the device.

To confirm that graphene was acting as an MFC anode rather than relying on the copper foil alone as an anode or as a complete galvanic cell, ${ }^{26}$ we fabricated two identical micro-sized MFCs and inserted copper foil, with and without graphene, as anodes. Both devices were operated under the same conditions, with the graphene anode outperforming the copper control by $>260 \%$ in power density and $>160 \%$ in current density (see Supplementary Material). As both anodes were made with the same copper foil, both would have experienced similar amounts of additional current from galvanic reactions, which does not diminish the difference in power and current values observed between the graphene anode and the copper control, but may exhibit slightly higher values in maximum power produced. ${ }^{5}$ The improved performance of the graphene device supported our assertion that graphene was acting as an MFC anode.

After establishing that saliva could be used to power micro-sized MFCs, we tested whether micro-sized MFCs could be operated with a more conventional organic fuel at similarly high organic concentrations. Other studies have found that power densities increase with higher organic contents but only at substrate concentrations of up to $2 \mathrm{gl}^{-1}$ (mass of the organic material). ${ }^{18,27}$ We therefore established two additional sets of identical graphene anode devices to test under high acetate loadings (20 and $10 \mathrm{gl}^{-1}$, measured as mass of sodium acetate and confirmed by concentrations of organic matter based on chemical oxygen demand). We inoculated the devices with the same wastewater source as the saliva devices, and then we began feeding the devices with the new acetate loadings, as shown in Figure 4a. As expected, the device fueled with $20 \mathrm{gl}^{-1}$ outperformed the one fueled with $10 \mathrm{gl}^{-1}$, with peaks at 490 and $300 \mathrm{~A} \mathrm{~m}^{-3}$, respectively. Power density curves were also measured $\left(8 \mathrm{Wm}^{-3}\right.$ for $\left.10 \mathrm{gl}^{-1}\right)$ and compared with those of saliva $\left(6 \mathrm{Wm}^{-3}\right)$ (Figure $\left.4 \mathrm{~b}\right)$. The microsized MFC that was fueled with $20 \mathrm{gl}^{-1}$ failed after 10 days due to clogging of the feeding syringes of the device by dried acetate powder. These results showed that high concentrations of non-conventional (saliva) and conventional (acetate) materials could serve as fuel sources in micro-sized MFCs, but further studies should also examine other organic sources to avoid reactor clogging.

\section{DISCUSSION}

In considering the use of saliva and the well-known positive correlations between solution conductivity and improved power generation in MFCs, a possible application of the micro-sized MFC is an ovulation predictor based on the conductivity of a woman's saliva. It has been found that 5 days before ovulation there is sharp decrease in the conductivity of saliva, most likely caused by a peak in estrogen and subsequent decrease in the electrolyte concentration in saliva. ${ }^{28}$ The MFC could be used to measure this change in 
conductivity to identify the fertility period of a woman, while simultaneously using the power generated to send the data to a readily available device, such as a smart phone. This application could thus help to maintain a woman's health, as well as help couples in better family planning in a non-invasive, easy-to-use method.

This study is a first step in utilizing saliva and other highly concentrated organic fuels to power bio-electronics and holds great potential for further applications in this field. By producing nearly $1 \mu \mathrm{W}$ in power, this saliva-powered, micro-sized MFC already generates enough power to be directly used as an energy harvester in microelectronic applications. With the emergence of the field of ultralow power chip-level biomedical electronics, devices able to operate at sub-microwatt power outputs are becoming a reality. ${ }^{29,30}$ For example, an electroencephalograph seizure detection system-on-chip integrating a novel circuit architecture that required $<1 \mu \mathrm{W}$ power was demonstrated, ${ }^{31}$ making our production of $0.9 \mu \mathrm{W}$ a competitive device for energy harvesting for such applications. Micro-sized MFCs are an interesting and practical energy harvesting option for health and environmental monitoring system-on-chips. An advantage of the MFC as an energy harvester is the ability to utilize abundant liquid fuel sources, complementing other widely known energy harvesters, such as photovoltaic, thermoelectric and piezoelectric systems, which use a different set of fuel resources. Future research on micro-sized MFCs should explore new methods to increase the power production of the MFC into the $\mathrm{mW}$ range, making it usable in a larger variety of applications. A recent study, based on assembling three MFCs in a series, demonstrated the ability to produce $100 \mu \mathrm{W}$ of power from individual reactors, which produced $80 \mu \mathrm{A}$ of current each. ${ }^{32}$ Improvements in the cathode size and efficiency could also increase power production. ${ }^{18}$ Additionally, creating a more efficient air cathode, one specifically designed for the micro-sized MFC, is essential for improving power production.

\section{CONFLICT OF INTEREST}

The authors declare no conflict of interest.

\section{ACKNOWLEDGEMENTS}

We would like to thank Professor Gary Amy, from KAUST, for providing us with laboratory space in the Water Desalination and Reuse Center; Dr Christiane Hoppe-Jones for help with liquid characterization; Daniah Assaadi, from KAUST, for taking the photographs; Shaiza Sinha and Mariam Mahmoud for help with the MFC assembly; and Olga Zausalina for helping with the preparation of the graphics.

Author contributions: $\mathrm{MMH}, \mathrm{JEM}$, and BEL conceived the experiments. MMH directed the experiments. JEM performed the experiments. RMQ synthesized and characterized the graphene. JEM and BEL analyzed the data. JEM and MMH co-wrote the manuscript. All authors discussed the results and helped with the preparation of the final manuscript.

1 Logan, B. Microbial Fuel Cells. 1st edn (John Wiley \& Sons, Inc., Hoboken, NJ, USA 2008).

2 Lovley, D. Bug juice: harvesting electricity with microorganisms. Nat. Rev. Microbiol. 4 , 497-508 (2006).

3 Qian, F. \& Morse, D. Miniaturizing microbial fuel cells. Trends Biotechnol. 29, 62-69 (2011).

4 Wang, H., Bernarda, A., Huang, C., Lee, D. \& Chang, J. Microsized microbial fuel cells: a mini-review. Bioresour. Technol. 102, 235-242 (2011).

5 Logan, B. E., Hamelers, B., Rozendal, R., Schroder, W., Keller, J., Freguia, S., Aelterman, P., Verstaete, W. \& Rabaey, K. Microbial fuel cells: methodology and technology. Environ. Sci. Technol. 40, 5181-5192 (2006).
6 Wei, J., Liang, P. \& Huang, X. Recent progress in electrodes for microbial fuel cells. Bioresour. Technol. 102, 9335-9344 (2011).

7 Alatraktchi, F., Zhang, Y., Noori, J. \& Angelidaki, I. Surface area expansion of electrodes with grass-like nanostructures and gold nanoparticles to enhance electricity generation in microbial fuel cells. Bioresour. Technol. 123, 177-183 (2012).

8 Logan, B., Cheng, S., Watson, V. \& Estadt, G. Graphite fiber brush anodes for increased power production in air-cathode microbial fuel cells. Environ. Sci. Technol. 41, 3341-3346 (2007).

9 Inoue, S., Parra, E. A., Higa, A., Jiang, Y., Wang, P., Buie, C. R., Coates, J. D. \& Lin, L. Structural optimization of contact electrodes in microbial fuel cells for current density enhancements. Sensor Actuat. A Phys. 177, 30-36 (2012).

10 Tsai, H. Y., Wu, C. C., Lee, C. Y. \& Shih, E. P. Microbial fuel cell performance of multiwall carbon nanotubes on carbon cloth as electrodes. J. Power Sources 194, 199-205 (2009).

11 Xie, X., Hu, L., Pasta, M., Wells, G. F., Kong, D., Criddle, C. S. \& Cui, Y. Three dimensional carbon nanotube-textile anode for high-performance microbial fuel cells. Nano Lett. 11, 291-296 (2011).

12 Mink, J. E., Rojas, J. P., Logan, B. E. \& Hussain, M. M. Vertically grown multiwalled carbon nanotube anode and nickel silicide integrated high performance microsized (1.25 $\mu \mathrm{L}$ ) microbial fuel cell. Nano Lett. 12, 791-795 (2012).

13 Gadhamshetty, V. \& Koratkar, N. Nano-engineered biocatalyst electrode structures for next generation microbial fuel cells. Nano Energy 1, 3-5 (2012).

14 Xiao, L., Damien, J., Luo, J., Jang, H., Huang, J. \& He, Z. Crumbled graphene particles for microbial fuel cell electrodes. J. Power Sources 208, 187-192 (2012).

15 Liu, J., Qiao, Y., Guo, C., Lim, S., Song, H. \& Li, C. Graphene/carbon cloth anode for high-performance mediatorless microbial fuel cells. Bioresour. Technol. 114, 275-280 (2012).

16 Yuan, Y., Zhou, S., Zhao, B., Zhuang, L. \& Wang, Y. Microbially-reduced graphene scaffolds to facilitate extracellular electron transfer in microbial fuel cells. Bioresour. Technol 116, 453-458 (2012).

17 Zhang, Y., Mo, G., Li, X., Zhang, W., Zhang, J., Ye, J., Huang, Xiaodan \& Yu, C. A graphene modified anode to improve the performance of microbial fuel cells. J. Power Sources 196, 5402-5407 (2011).

18 Cheng, S. \& Logan, B. E. Increasing power generation for scaling up single-chamber air cathode microbial fuel cells. Bioresour. Technol. 102, 4468-4473 (2011).

19 Cheng, S., Liu, H. \& Logan, B. Increased performance of single-chamber microbial fuel cells using an improved cathode structure. Electrochem. Commun. 8, 489-494 (2006).

20 Hou, H., Li, L., De Figueiredo, P. \& Han, A. Air-cathode microbial fuel cell array: a device for identifying and characterizing electrochemically active microbes. Biosens. Bioelectron. 26, 2680-2684 (2011).

21 Chen, Y., Zhao, Y., Qui, K., Chu, J., Lu, R., Sun, M., Liu, X., Sheng, G., Yu, H., Chen, J., Li, W., Liu, G., Tian, Y. \& Xiong, Y. An innovative miniature microbial fuel cell fabricated using photolithography. Biosens. Bioelectron. 26, 2841-2846 (2011).

22 Lovley, D. R. \& Phillips, E. J. P. Novel mode of microbial energy metabolism: organic carbon oxidation coupled to dissimilatory reduction of iron or manganese. Appl. Environ. Microbiol. 54, 1472-1480 (1988).

23 Chiappin, S., Antonelli, G., Gattie, R. \& De Palo, E. F. Saliva specimen: a new laboratory tool for diagnostic and basic investigation. Clin. Chim. Acta 383, 30-40 (2007).

24 Yong, Y., Dong, X., Chan-Park, M., Song, H. \& Chen, P. Macroporous and monolithic anode based on polyaniline hybridized three-dimensional graphene for high-performance microbial fuel cells. ACS Nano 6, 2394-2400 (2012).

25 Matsuda, Y., Deng, W. Q. \& Goddard, W. A. III Contact resistance properties between nanotubes and various metals from quantum mechanics. J. Phys. Chem. $111,11113-11116$ (2007).

26 Logan, B. E. Essential data and techniques for conducting microbial fuel cell and other types of bioelectrochemical system experiments. Chem. Sus. Chem. 5, 988-994 (2012).

27 Min, B. \& Logan, B. E. Continuous electricity generation from domestic wastewater and organic substrates in a flat plate microbial fuel cell. Environ. Sci. Technol. 38, 5809-5814 (2004).

28 Huang, Z., Wu, Z., Zhou, F. \& Zhou, J. Ovulation prediction by monitoring the conductivity of woman's saliva. IEEE/EMBS Proc. 5, 2344-2346 (1997).

29 Sarpeshkar, R. Ultra Low Power Bioelectronics: Fundamentals, Biomedical Applications, and Bio-inspired Systems (Cambridge University Press, Cambridge, MA, USA, 2010).

30 Chandrakasan, A., Verma, N. \& Daly, D. Ultralow-power electronics for biomedical applications. Annu. Rev. Biomed. Eng. 10, 247-274 (2008).

31 Sridhara, S., Direnzo, M., Lingam, S., Lee, S., Blazquez, R., Maxey, J., Ghanem, S., Lee, Y., Abdallah, R., Singh, P. \& Goel, M. Microwatt embedded processor platform for medical system-on-chip applications. Symp. VLSI Circuits 15-16 (2010).

32 Choi, S. \& Chae, J. An array of microliter-sized microbial fuel cells generating $100 \mu \mathrm{W}$ of power. Sensor Actuat. A Phys. 177, 10-15 (2012).

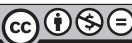

This work is licensed under a Creative Commons Attribution-NonCommercial-NoDerivs 3.0 Unported License. To view a copy of this license, visit http://creativecommons. org/licenses/by-nc-nd/3.0/ 